Trauma Berufskrankh 2017· 19 (Suppl 2):S126-S134 DOI 10.1007/s10039-016-0225-7

Online publiziert: 10. Januar 2017

๑) Springer Medizin Verlag Berlin 2016

CrossMark

J. Frank - A. L. Sander · M. Voth - I. Marzi

Klinik für Unfall-, Hand- und Wiederherstellungschirurgie, Universitätsklinikum Frankfurt, GoetheUniversität, Frankfurt am Main, Deutschland

\title{
Sekundäre Rekonstruktionen am Ellenbogengelenk im Kindesalter
}

komplexe Gelenksituation mit 3 aufeinander abgestimmten Gelenken präzise stimmen, um eine gute Beweglichkeit in alle Richtungen zu erlauben. Heutzutage beruhen Fehlstellungen im Bereich des Ellenbogens meist auf diagnostischen und therapeutischen Schwierigkeiten bei der Primärversorgung. Hier wird zum einen in der Diagnostik die Relevanz der Verletzung unterschätzt, oder zum anderen gelingt die optimale Versorgung nicht [1]. Die relevanten Verletzungen am Ellenbogen sind in - Tab. 1 aufgeführt. Besonders hervorzuheben sind dabei die suprakondyläre Oberarmfraktur, die Condylus-radialis-Fraktur und die Monteggia-Läsion mit Luxation des Speichenkopfes. Aus diesem Grund werden diese Frakturen nach von Laer zu den „Kadiläsionen“ gezählt, da sie bei unzureichender Behandlung oftmals rechtliche Konsequenzen haben [2-4]. Das Wissen um die Problematik hat in den letzten Jahren zu einem strukturierten Fortbildungsangebot geführt. Mit der Änderung der Anforderungen der gesetzlichen Unfallversicherung zur Beteiligung am Durchgangsarztverfahren in der Fassung vom 01.01.2011 wurde für einen 5-Jahres-Zeitraum eine Fort- bildung im Bereich Kindertraumatologie verpflichtend.

\section{Deformitäten des distalen Humerus}

Die bekannten Deformitäten des distalen Humerus und dessen Ursachen sind in - Tab. 2 aufgelistet. Die häufigste Deformität ist die suprakondyläre Humerusfraktur. Aus der multizentrischen Sammelstudie der Deutschen Gesellschaft für Unfallchirurgie wissen wir, dass die Ergebnisse gut sind $[5,6]$. Es findet sich jedoch bei den höheren Schweregraden (III und IV) auch ein deutlicher Anteil - nahezu $20 \%$ - an schlechten Ergebnissen (•Tab. 3; [2]). Die mittlerweile weitverbreitete Schulung und die damit verbundenen Kenntnisse haben sicherlich die Anzahl an korrekten Repositionen und adäquaten Stabilisierungen gesteigert. Eine relevante Varusfehlstellung findet sich daher nur noch in maximal $3 \%$ der Fälle, und man kann die Zahlen bis $60 \%$ Fehlstellungen, basierend auf einem konservativen Therapieregime, als historisch betrachten [7-9]. Valgusfehlstellungen basieren meist auf Läsionen im Bereich des Condylus radialis und
Tab. 1 Relevante kindliche Ellenbogenverletzungen [4]

Suprakondyläre Humerusfraktur $\quad 6,5 \%$

Condylus-radialis-Fraktur $\quad 1,8 \%$

Ellenbogenluxation und Epicon- $\quad 1,3 \%$

dylus ulnaris

Radiuskopffraktur $\quad 1,3 \%$

Monteggia-Verletzung $\quad 1,3 \%$

Olekranonverletzung $\quad 0,5 \%$
Tab. 2 Posttraumatische Deformitäten des distalen Humerus [4]

\begin{tabular}{|c|c|}
\hline Deformitäten & Ursachen \\
\hline \multirow[t]{3}{*}{ Cubitus varus } & Hauptsächlich nach suprakondylären Frakturen \\
\hline & Ulnarer Fugenverschluss \\
\hline & Radiale Stimulation - Kondylusfraktur \\
\hline \multirow[t]{2}{*}{ Cubitus valgus } & Radiale Pseudarthrose - Kondylusfraktur \\
\hline & Ulnare Stimulation - Kondylusfraktur \\
\hline \multirow[t]{2}{*}{ Pseudarthrose } & Radiale Kondylusfraktur \\
\hline & Ulnare Epikondylusfraktur \\
\hline Fischschwanzdeformität & Zentraler Verschluss der Fuge - Kondylusfraktur/Y-Fraktur \\
\hline
\end{tabular}



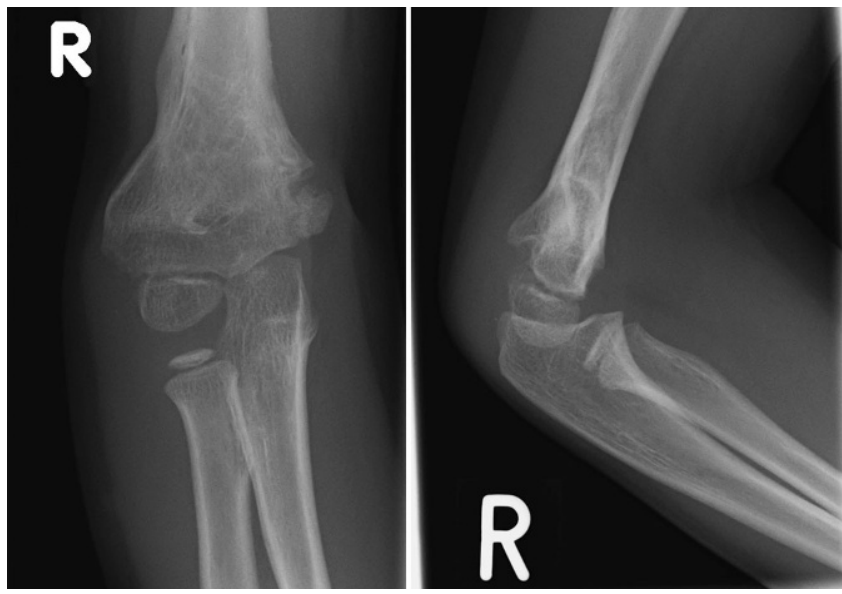

Abb. 1 ॥ Röntgenbild einer fehlverheilten, suprakondylären Humerusfraktur mit Cubitus varus
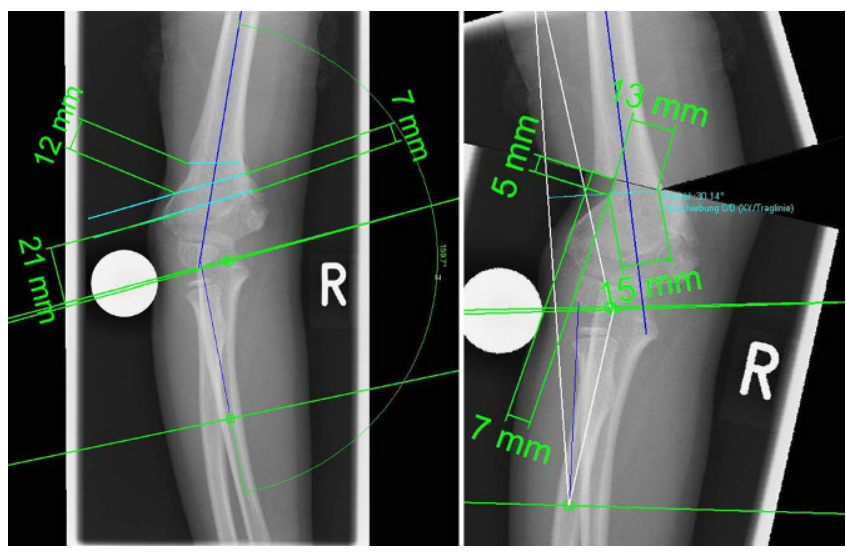
mie
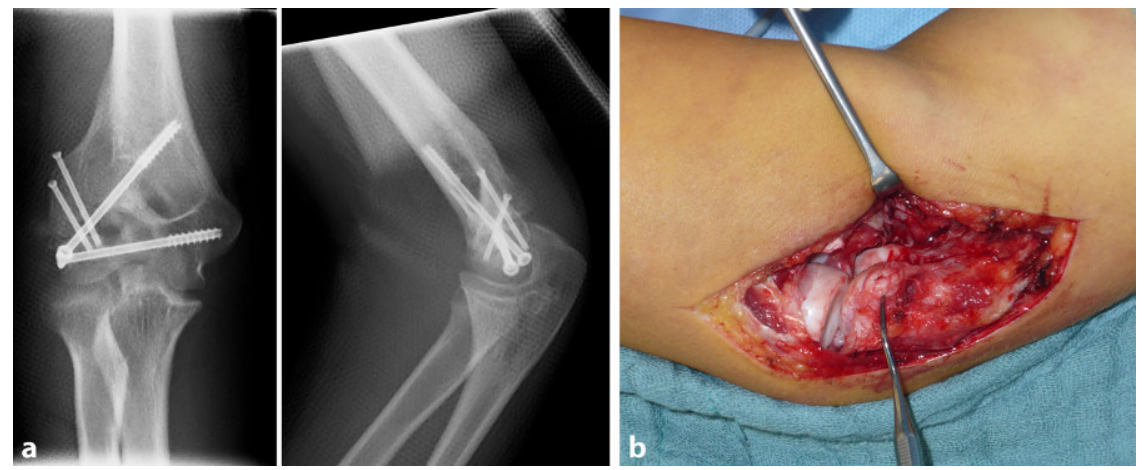

Abb. $4 \Delta$ a Röntgenbild des distalen Humerus nach Schraubenosteosynthese der Condylus-radialis-Pseudarthrose. $\mathbf{b}$ Intraoperativer Befund

führen hin und wieder auch zu Pseudarthrosen in diesem Bereich.

\section{Therapie}

\section{Grundlagen}

Gelingt die Einstellung und Fixierung des Gelenkblockes am distalen Humerus unzureichend, dann resultiert meist eine mehrdimensionale Fehlstellung mit Varusdeformität, Innenrotationsfehler und Antekurvation (• Abb. 1). Eine Indikation zur Korrektur ergibt sich aus Funktionsstörungen, dem kosmetischen Erscheinungsbild und gelegentlichen Nervenirritationen. Die Valgusdeformität tritt weit weniger in Erscheinung, und daher sind korrigierende Eingriffe
Abb. $3<$ Röntgenbild und digitale Planung vor der Durchführung der korrigierenden Osteoto-

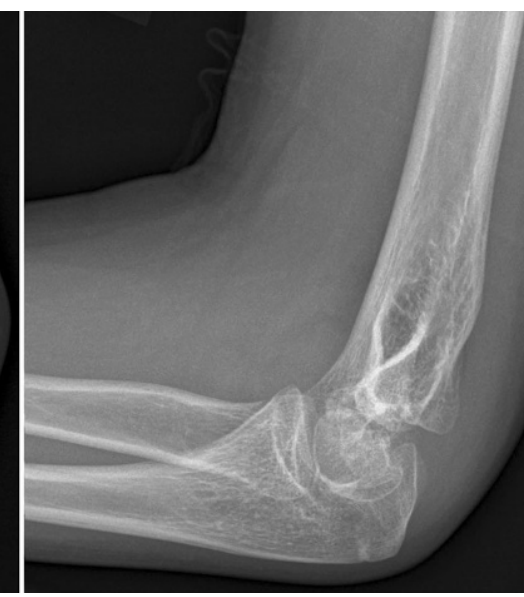

Abb. 2 ॥ Röntgenbild des distalen Humerus. Pseudarthrose des Condylus radialis mit Valgusdeformität

selten, zumal hier das Erscheinungsbild und die Funktion weniger beeinträchtigt sind. Probleme, die gelegentlich eine Korrektur indizieren, sind mediale Bandinstabilitäten bzw. Überlastungen und eine damit verbundene Irritation des N. ulnaris. Kommt es nach einer Condylus-radialis-Fraktur zu einer Pseudarthrosebildung, ist meist eine operative Therapie notwendig, um die damit meist verbundene Instabilität der radialen Säule zu beseitigen ( $\bullet$ Abb. 2).

\section{Operative Therapie}

\section{Präoperative Vorbereitung}

Zur Vorbereitung gehört zunächst die exakte Erfassung der Deformität mit Bestimmung der Abweichung, Messung der Bewegung und Überprüfung der Gelenkfunktion bzw. Stabilität. Gerade die häufige Deformität nach suprakondylärer $\mathrm{Hu}$ merusfraktur führt bei Antekurvation zu einer Beuge- und bei Retrokurvation zu einer Streckhemmung.

Zusätzlich empfiehlt sich bei Hinweisen auf eine Nervenirritation dies ggf. messtechnisch zu verifizieren. Hier muss besonders auf die Irritation des N. ulnaris bei einer extremen Valgusdeformität hingewiesen werden, was eine Operationsindikation darstellen kann. Die weitere Behandlung und die alternativen Optionen müssen dann mit dem Kind und den Eltern im Detail besprochen werden und bei gegebener Operationsindikation die einzelnen Schritte - ggf. mit Abweichungen/zusätzlichen 
Maßnahmen. Das Kind muss ggf. bei aufwendigen Verfahren - wie z. B. einem Fixateur - besonders beachtet werden und eine sorgfältige Situationsbeschreibung, dem Alter entsprechend, erfahren. Hilfreich kann die Anwendung verschiedener Planungsprogramme sein. Hier können bei optimierter Kalibrierung Abmessungen z. B. der Osteotomie zur Gelenklinie, der Keilgröße, das Ausmaß der Verschiebung/Translation entsprechend der gewünschten Achskorrektur bestimmt werden (-Abb. 3). Eine solche Planung - auch als Zeichnung - hilft sehr, sich bereits präoperativ mit den ggf. auftretenden Problemen und Varianten auseinanderzusetzen.

\section{Operative Methoden}

Pseudarthrosen, wie sie meist im $\mathrm{Zu}$ sammenhang mit Läsionen des Condylus radialis gesehen werden, sollten zur Wiedererlangung der Stabilität verschraubt werden. Eine damit verbundene Knochenentnahme bzw. Spongiosaplastik muss individuell vom Defekt bzw. vom Ausmaß der Distalisierung zur Kompensation der Valgusdeformität abhängig gemacht werden (• Abb.4). In manchen Fällen reicht die alleinige Verschraubung nach Anfrischung der Pseudarthrose.

Die operationstechnische Vorgehensweise zur Korrektur einer Valgus- bzw. Varusdeformität richtet sich nach dem Alter des Kindes. Es muss dabei zwischen Osteosynthesetechniken und Korrekturtechniken zur Osteotomie unterschieden werden. Bei Adoleszenten und nahe dem Wachstumsabschluss kann entsprechend der Korrektur beim Erwachsenen eine stabile Doppelplattenosteosynthese erfolgen (- Abb. 5) oder mittels spezieller Y-Platten behandelt werden [10]. Für die jüngeren Kinder wird zur Stabilisierung - abhängig von der Korrekturtechnik die Kirschner-Drahtosteosynthese oder der Fixateur externe verwendet.

Von der Osteotomietechnik ist vermutlich die radialseitige Closed-Wedgebzw. French-Osteotomie - nach dem Erstbeschreiber - technisch am einfachsten und wird heute meist mittels Fixateur in Kombination mit einer Kirschner-Drahtosteosynthese durchgeführt [11]. Insbesondere bei kleinen Kindern wurde, ähnlich einer Pendel-

Trauma Berufskrankh 2017 · 19 (Suppl 2):S126-S134 DOI 10.1007/s10039-016-0225-7

(c) Springer Medizin Verlag Berlin 2016

J. Frank · A. L. Sander $\cdot$ M. Voth · I. Marzi

\section{Sekundäre Rekonstruktionen am Ellenbogengelenk im Kindesalter}

\section{Zusammenfassung}

Es ist bekannt, dass es im Bereich des Ellenbogens bei Kindern und Jugendlichen häufig zu Verletzungen kommt. Der geringe Anteil am Längenwachstum im Bereich des distalen Humerus und proximalen Unterarms trägt dazu bei, dass die Spontankorrektur limitiert und frühzeitig in jungem Alter ausgeschöpft ist. Probleme bei der Primärversorgung sind häufig die Ursache für posttraumatische Deformitäten, die dann gelegentlich eine sekundäre Korrektur erfordern. Besonders hervorzuheben sind die Folgen nach suprakondylärer Humerusfraktur mit Cubitus varus, seltener Cubitus valgus, Fehlstellung bzw. Pseudarthrose des Condylus radialis und eine persistierende Luxation des Radiuskopfes, verursacht durch eine Monteggia-Läsion. Die Korrektur dieser Deformitäten ist anspruchsvoll, und es werden in der Literatur hohe Komplikationsraten angegeben. Unabhängig davon muss entsprechend der Verletzung das zu erwartende Funktionsdefizit abgeschätzt und bei kritisch zu stellender, aber gegebener Korrekturindikation diese zügig durchgeführt werden, bevor es durch die Fehlstellung zu einer sekundären Gelenkbzw. Knochenumformung kommt.

\section{Schlüsselwörter}

Korrekturosteotomie · Cubitus varus · Cubitus valgus · Condylus radialis · Monteggia-Läsion

\section{Secondary reconstruction of the elbow joint in childhood}

\section{Abstract}

It is well known that fractures in the region of the elbow are very common in children and adolescents. The small contribution to physical growth by the distal humerus and proximal forearm is the reason that spontaneous correction is limited and exhausted at an early age. Difficulties in primary care and fixation are often the reasons for posttraumatic deformities, which occasionally then necessitate secondary correction. Particularly emphasized are the sequelae of supracondylar humeral fractures with cubitus varus and less frequently cubitus valgus, malalignment or pseudarthrosis of the radial condyle and a persistent luxation of the radial head caused by Monteggia's fractures. Correction of such deformities is very demanding and high complication rates are described in the literature; however, the expected functional deficiencies must be estimated and if critically indicated a corrective procedure must be performed in a timely manner. This should avoid secondary joint and bone reshaping which would make correction more difficult or even impossible.

\section{Keywords}

Corrective osteotomy · Cubitus varus · Cubitus valgus - Radial condyle · Monteggia's fracture osteotomie am Tibiakopf, die DomeOsteotomie propagiert. Dieser Methode wurde in der Literatur eine etwas höhere Komplikationsrate angelastet [12-14]. Eine weitere Modifikation stellt die StepCut-Osteotomie dar, hier werden durch die Entnahme eines Dreieckkeils am distalen Humerus eine Rotation wie auch eine Medialisierung ermöglicht. Dadurch kann zum einen die unschöne radiale Prominenz des Knochens reduziert und zum anderen die Stabilität durch die Fragmenteinpassung verbessert werden [10]. Aus dem gleichen Grund und wegen der sichtbaren
Narbenbildung propagieren manche Autoren die Korrektur über den medialen Zugang ggf. auch als Open-Wedge-Osteotomie und führen hier die sichtbaren radialseitigen Narben als besonders störend an. Die Stabilisierung erfolgt meist durch einen Fixateur externe und kann auch im Sinne einer Kallusdistraktion durchgeführt werden [15-19].

Das von uns bevorzugte Verfahren entspricht der Methode nach Linhart und Kraus [20] und wird mittels Fixateur externe und dreidimensionaler suprakondylärer Derotationsosteotomie durchgeführt. Entsprechend der präoperativen 

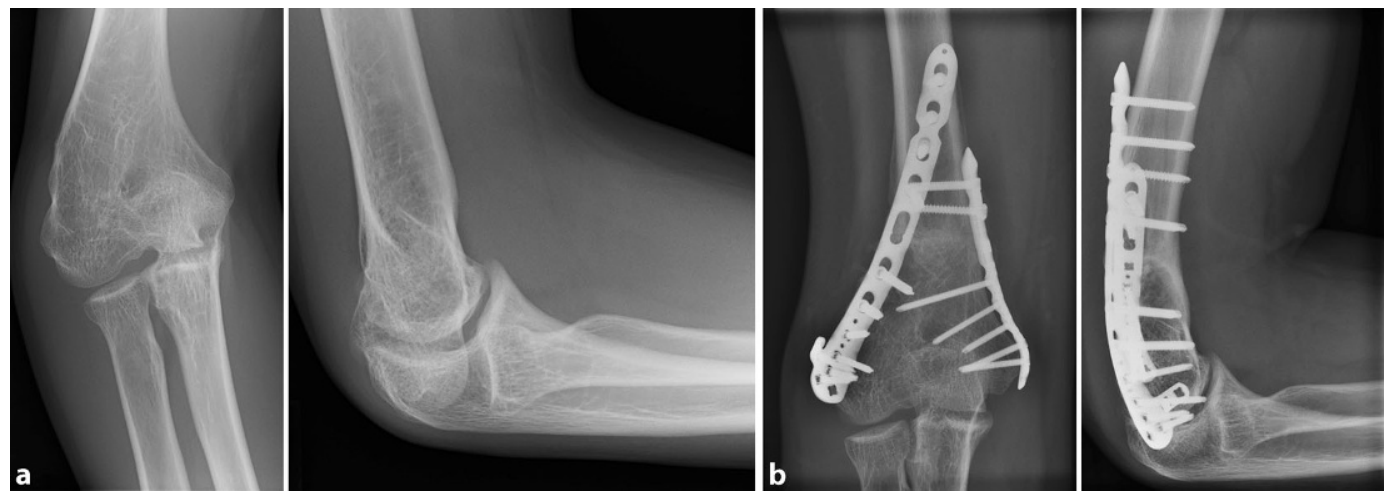

Abb. $5<$ Varusdeformität nach distaler Humerusfraktur. a Präoperative Röntgenaufnahme. b Postoperatives Röntgenbild nach Korrektur und Stabilisierung mittels Doppelplattenosteosynthese
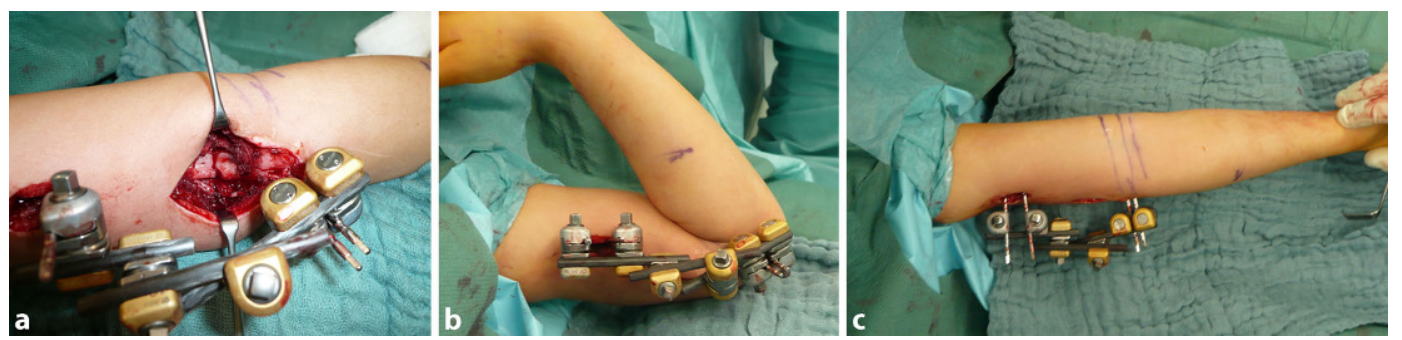

Abb. $6<$ Intraoperatives Bild der Korrektur einer Varusdeformität nach suprakondylärer Humerusfraktur. a Blick auf die Osteotomie. b Funktionskontrolle mit Flexion und $\mathrm{c}$ Extension
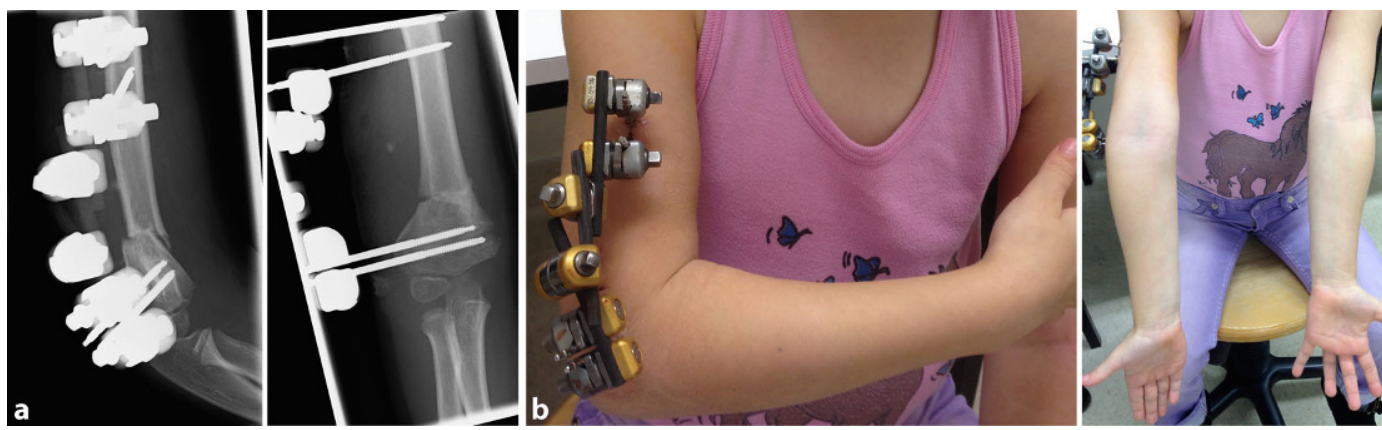

Abb. $7<$ Postoperative Bilder nach Korrektur eines Cubitus varus. a Postoperative Röntgenbilder. b Klinische Funktion

Planung (• Abb. 3) erfolgt die Implantation der Fixateurpins. Die primär eingebrachten distalen Pins liegen proximal der Wachstumsfuge, die proximalen Pins werden über eine offene Darstellung des Humerus eingebracht, um sicher eine Läsion des N. radialis zu verhindern. Nach entsprechender Probemontage erfolgt dann die Osteotomie. Bei der Planung sollte darauf geachtet werden, die Osteotomie möglichst nahe am CORA („center of rotation and angulation“) $\mathrm{zu}$ machen. Bei Abweichungen muss die resultierende Translation mit bedacht werden. Dies gilt insbesondere dann, wenn bei offenen Wachstumsfugen die distalen Pins weiter proximal platziert werden müssen. Dann erfolgen die Osteotomie und freie Einstellung des distalen Gelenkblockes entsprechend der Fehlstellung (-Abb. 6a-c). Es ist zu bemerken, dass der Fixateur externe im Allgemeinen von den Kindern gut toleriert wird. Nach entsprechender Pflegeanleitung kann der Ellenbogen so weit wie möglich bewegt werden (• Abb. 7).

\section{Deformitäten des proximalen Unterarmes}

Im Bereich des proximalen Unterarms müssen die Radiuskopfdeformitäten z. B. nach Radiushalsfrakturen oder die Varusdeformität der Elle nach einer Olekranonfraktur erwähnt werden. Besonders $\mathrm{zu}$ beachten ist die Radiuskopfluxation nach Monteggia-Läsion. Die Läsion findet sich in verschiedenen Formen und kann sowohl nach Bado entsprechend der Läsion bei Erwachsenen oder nach Letts et al., der diese dem kindlichen Verletzungsmuster angepasst hat [21-23], klassifiziert werden. Hier wird besonders auch das Ulna-Bowing mit einbezogen, was ohne offensichtliche Fraktur das Erkennen der Radiuskopfluxation besonders erschwert (• Abb. 8; [24]).

Eine chronische Luxation kann $\mathrm{zu}$ schwerwiegenden Gelenkveränderungen führen, und das Erkennen in der Primärphase ist hier besonders wichtig. Daher gelten folgende 3 Regeln:

1. Bei jeder Ulnaschaftfraktur (auch Bowing-Fraktur) soll der Ellbogen in 2 Ebenen geröntgt werden bzw. umgekehrt der Unterarm bei Radiuskopfluxation.

2. Auf jedem Ellenbogenbild und in jeder Ebene muss sich der Radiuskopf auf den Capitulumkern zentrieren.

3. Bei Verdacht auf kongenitale Luxation (kein adäquates Trauma) erfolgt ein Seitenvergleich (Unterarm, Handgelenk). 
Tab. 3 ScorezurEinteilung des Behandlungsergebnisses nach suprakondylärer Humerusfraktur [4]

\begin{tabular}{|c|c|c|}
\hline Punkte & Radiologisches Ergebnis & Klinisches Ergebnis \\
\hline $0=$ ideal & Keine Achsabweichung a.-p., seitlich gut & $\begin{array}{l}\text { Kein Funktionsdefizit, Achsen } \\
\text { seitengleich }\end{array}$ \\
\hline $1=$ gut & $\begin{array}{l}\text { Bis } 5^{\circ} \text { Varus/Valgus, Ante-, Rekurvations- } \\
\text { fehler bis } 10^{\circ}\end{array}$ & $\begin{array}{l}\text { Bis } 10^{\circ} \text { Defizit Extension/Flexion, } \\
\text { Achsfehlstellung bis } 5^{\circ}\end{array}$ \\
\hline $2=$ tolerabel & $5-10^{\circ}$ Varus/Valgus & $\begin{array}{l}10-20^{\circ} \text { Defizit Extension/Flexion } \\
\text { Valgus und Varus } 5-10^{\circ}\end{array}$ \\
\hline $3=$ schlecht & $10^{\circ}$ Varus-Valgus oder mehrere Fehler & $\begin{array}{l}20^{\circ} \text { Differenz in einer Ebene oder } \\
\text { mehr }\end{array}$ \\
\hline
\end{tabular}

Die akute Luxation ist vergleichsweise einfach operativ zu versorgen, wohingegen die chronische Luxation mit Fehlheilung ein sehr differenziertes Vorgehen sowohl diagnostisch wie auch korrekturtechnisch erfordert. Zudem werden durch die Weichteilirritation bei der Radiuskopffehlstellung bis zu $10 \%$ Nervenläsionen berichtet $[2,7,25,26]$.

\section{Therapie}

\section{Grundlagen}

Wesentlich für die Therapieplanung bei einer chronischen Radiuskopfluxation bzw. Monteggia-Läsion ist der Zeitpunkt der Luxation, da es im Verlauf $\mathrm{zu}$ weiteren Verformungen kommen kann, die eine optimale Rekonstruktion verhindern oder gar unmöglich machen. Bei der akuten Luxation sollten die entscheidenden Gelenkstrukturen unverändert sein, während bei einer veralteten posttraumatischen chronischen Luxation erhebliche Deformitäten vorliegen können, unabhängig davon, ob nun rein kongenital oder entwicklungsbedingt. Die typischen Merkmale der kongenitalen Dislokation sind in • Tab. 4 aufgelistet. An chronischen Veränderungen müssen vor einer Korrektur die in - Tab. 5 aufgeführten Deformierungen beachtet werden, da sie möglicherweise eine erfolgreiche Korrektur verhindern (- Abb. 9 und 10). Prinzipiell kann eine Korrekturindikation erwogen werden bei:

- Instabilität,

- Irritation des N. ulnaris,
- Bewegungseinschränkung/ Beeinträchtigung des Ellenbogens,

- Progression der Deformität,

- Schmerzen,

- kosmetischen Beeinträchtigungen.

\section{Operative Therapie}

\section{Präoperative Vorbereitung}

Entsprechend der Ausgangssituation müssen die Schritte zur Korrektur möglichst exakt mit dem Kind und den Eltern besprochen werden. Die Ausgangsfunktion muss erfasst werden, und die Chancen, bestehende Funktionsbehinderungen zu beheben, müssen abgeschätzt werden. Es muss ggf. besprochen werden, dass nur Teilziele erreichbar sind. Oft ist das primäre Ziel bei der Korrektur einer Monteggia-Läsion die Verbesserung der Beugung, da bei der typischen Form (- Abb. 8a-c) der akuten Verletzung der Radiuskopf meist nach ventral luxiert ist und damit die Flexion des Ellenbogens behindert (• Abb. 8).

\section{Operative Methoden}

Die Korrektur bei einer rechtzeitigen Diagnosestellung - etwa den ersten 6 bis 12 Wochen - gestaltet sich aufgrund der noch unwesentlichen Folgeverformung meist relativ gutmütig. Oft kann durch eine angulierende Osteotomie mit offener Einstellung des Radiuskopfes und Kontrolle in allen Bewegungsebenen die Situation beherrscht werden. Am Therapieende ist mit einer guten Funktion $\mathrm{zu}$ rechnen (• Abb. 11a-f).

Bei einer länger zurückliegenden Radiuskopfluxation muss neben den in - Tab. 5 aufgeführten Deformierungen

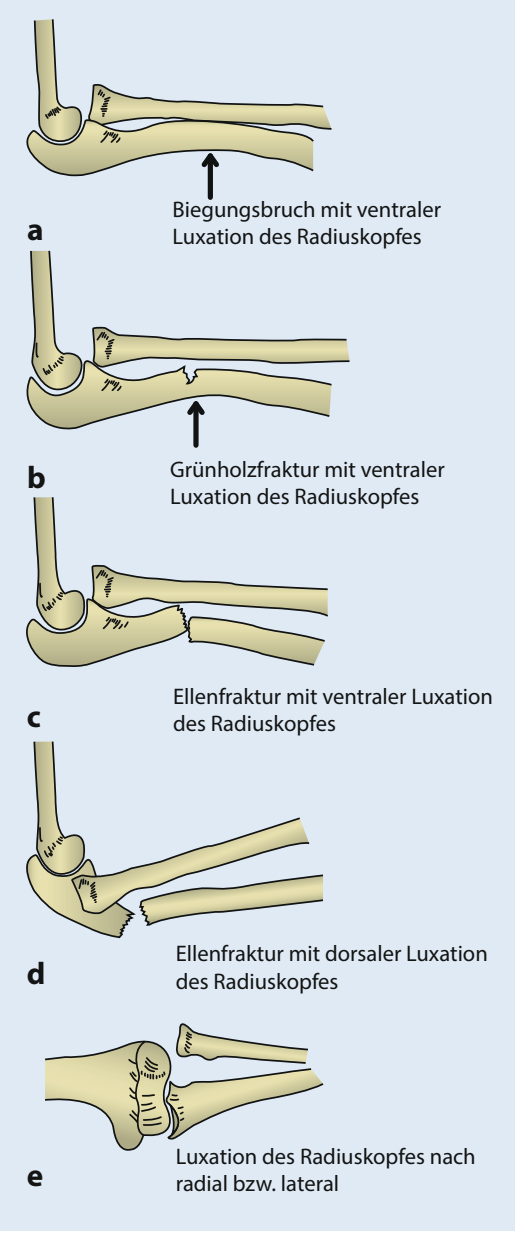

Abb. 8 ॥ Klassifikation der kindlichen Monteggia-Läsion. Modifikation der Bado-Klassifikation nach Letts et al. (Nach [22])

und der Ventralluxation des Radiuskopfes auch dessen Proximalisierung beachtet werden. Dadurch ist eine direkte Einstellung nahezu unmöglich, da hierfür eine extreme Angulation notwendig wäre und diese auch noch zusätzlich die Elle in Relation zur Speiche verkürzt $[22,25,27,28]$. Eine $\mathrm{zu}$ forcierte Einstellung kann zu einem übermäßigen Druck im Humeroradialgelenk und dadurch Verformungen oder sogar einer Osteonekrose führen. Eine Reluxation ist dabei ebenfalls wahrscheinlicher [29-31].

Um diese Probleme zu überwinden und eine Gelenkeinstellung zu erreichen, ist zunächst eine Distalisierung des Radiuskopfes notwendig. Dies wird mittels Ulnaosteotomie und Verlängerung durch Kallusdistraktion erreicht. Damit im Handgelenkbereich eine Störung ver- 


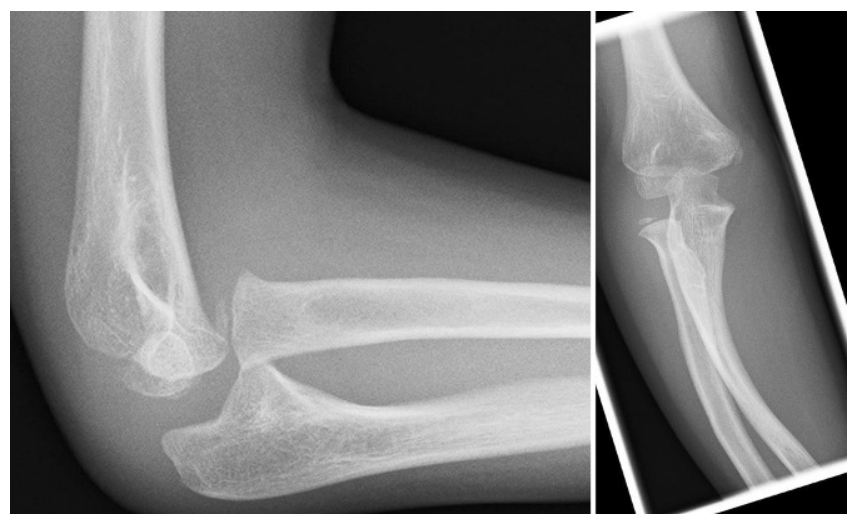

Abb. $9 \Delta$ Chronische posttraumatische Deformität der proximalen Elle mit radial konvexer Deformität und Luxation des Radiuskopfes aus dem Humeroradialgelenk
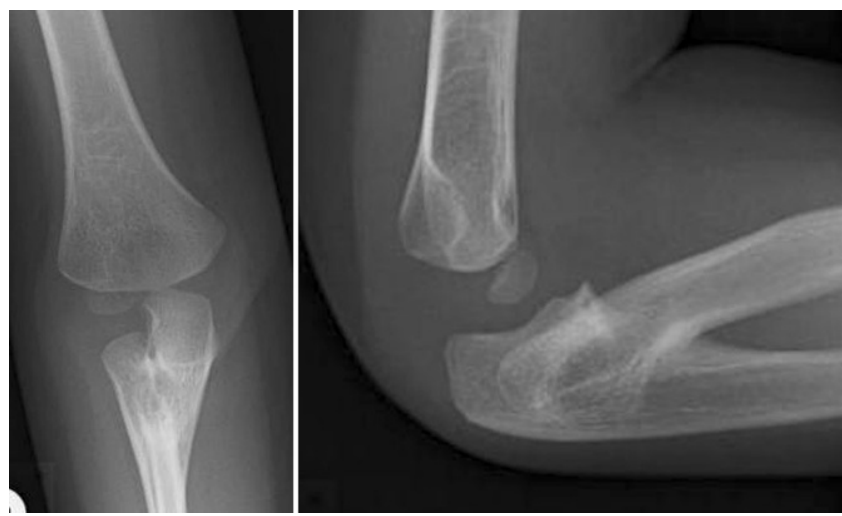

Abb. $10 \Delta$ Kongenitale Deformität des proximalen Radius mit Synostosebildung

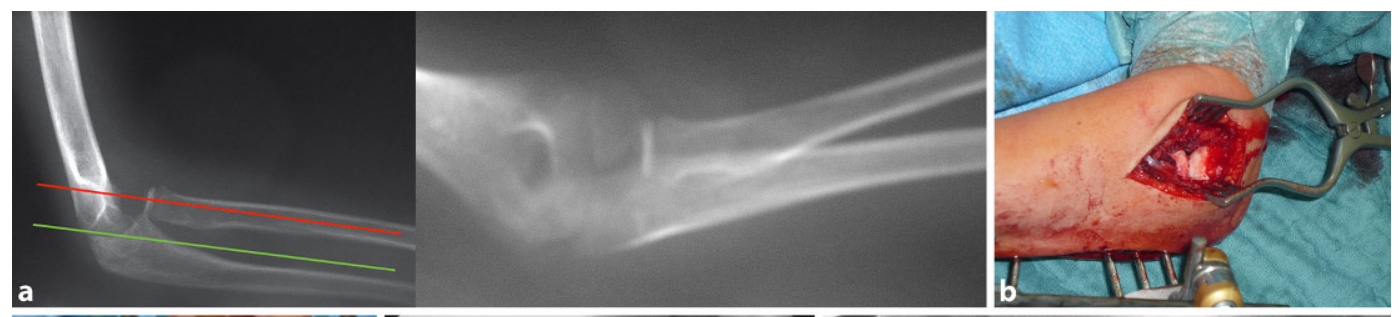

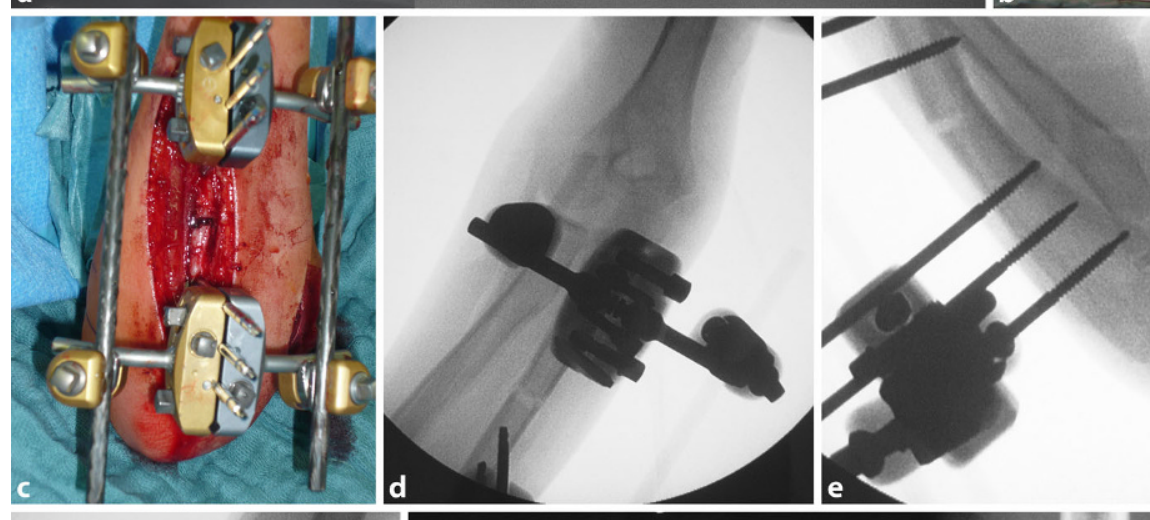
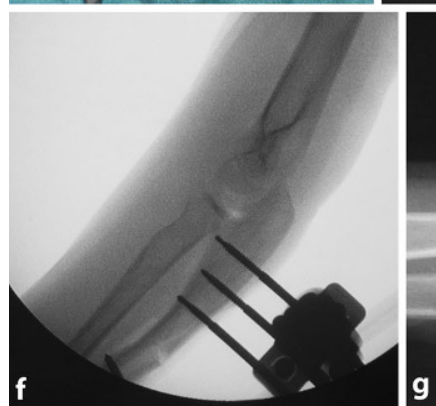
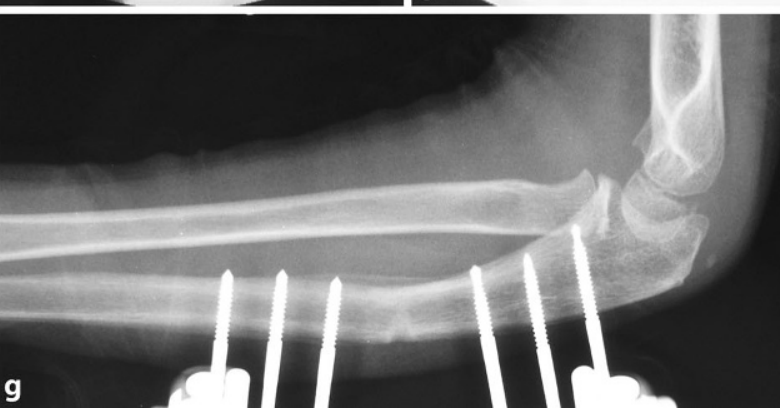

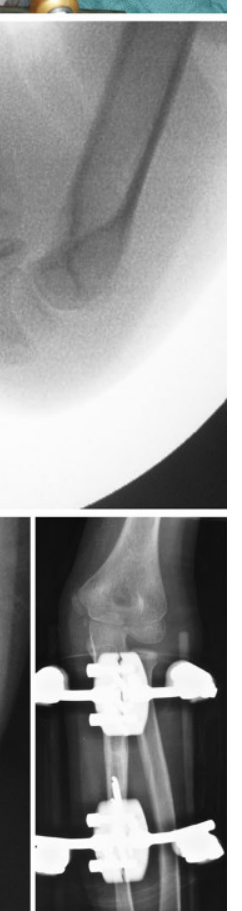

Abb. $11<$ Korrektur einer Monteggia-Läsion mit Radiuskopfluxation ohne wesentliche Deformität oder Proximalisierung des Radiuskopfes. a Präoperatives Röntgenbild: Die rote Linie markiert das Ausmaß der Ventralluxation, die grüne Linie wäre die zu erwartende Position. b Offene Einstellung des Radiuskopfes. c Klinisches Bilder der Angulation. d-f Intraoperative Röntgenkontrolle in verschiedenen Ebenen. g Ausheilungsbild mieden wird bzw. kein relativer Ulnavorschub provoziert wird, muss bei der Verlängerung die Speiche distal mit fixiert und gemeinsam mit dem körperfernen Anteil der Elle nach distal transportiert werden. Es sollte dabei ein Abstand vom proximalen Radiuskopf bis zum Capitulum von $\mathrm{ca} .1,5 \mathrm{~cm}$ erreicht werden.
Nach einer Ruhephase von etwa 3 Wochen erfolgen dann die Angulation und offene Einstellung wie zuvor beschrieben. Ein Verfahrenswechsel auf eine Plattenosteosynthese kann den Komfort verbessern und die lange Tragedauer des Fixateurs vermeiden (• Abb. 12a-f; [26, 32-38]). Während der gesamten Behand- lungsphase sollte - soweit möglich - eine Übungsbehandlung des Ellenbogens ohne Belastung erfolgen.

\section{Komplikationen}

Bei korrigierenden Eingriffen am Ellenbogen finden sich zunächst akute 

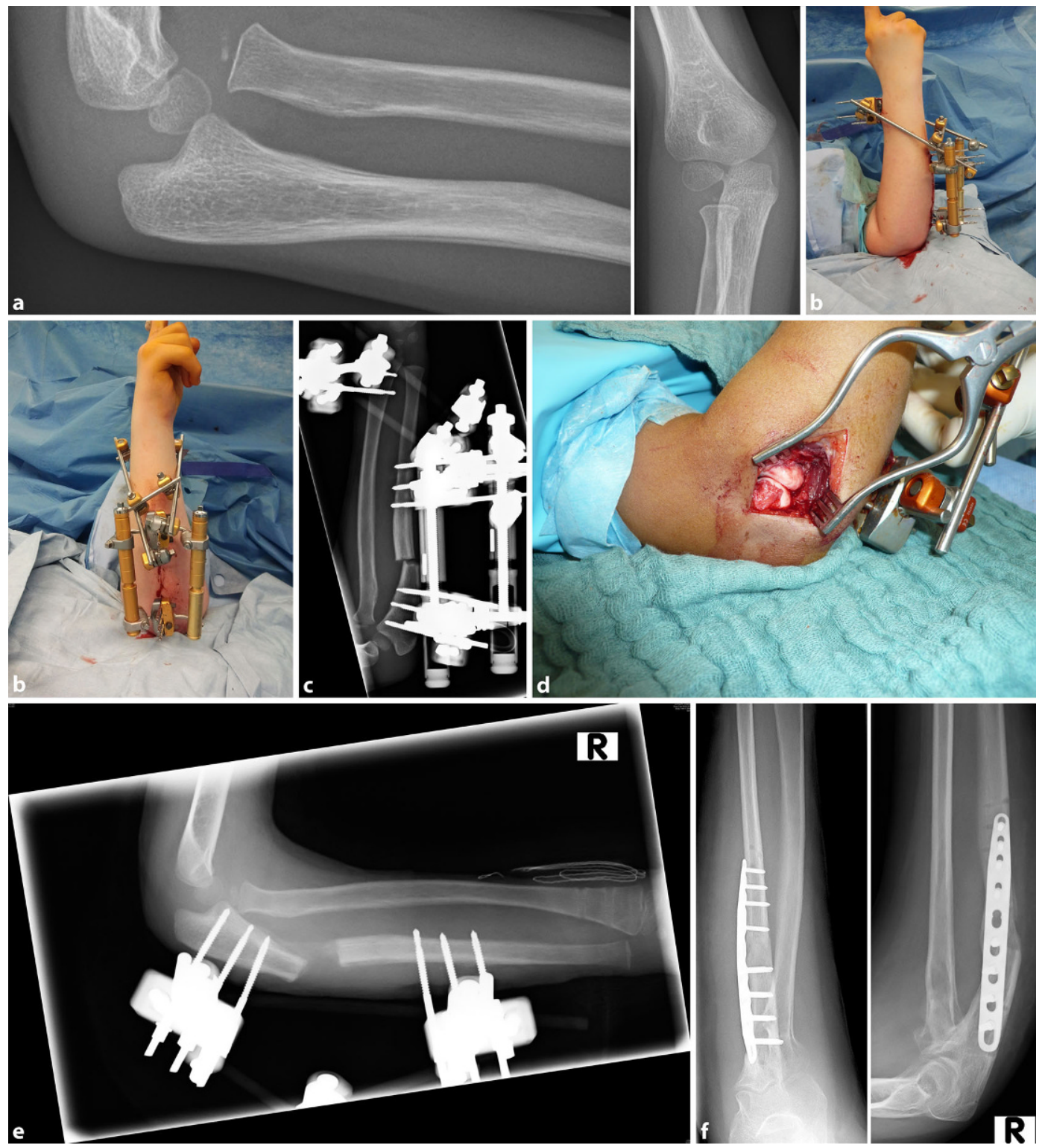

Abb. $12 \triangleleft$ Korrektur einer chronischen Monteggia-Läsion mit Radiuskopfluxation und deutlicher Proximalisierung. a Präoperatives Röntgenbild. b Intraoperatives Erscheinungsbild einer möglichen Fixateurmontage zur Korrektur (Hoffmann II Compact System der Fa. Stryker). c Röntgenverlaufskontrolle der Kallusdistraktion. d Offene Einstellung und Fixateurumbau. e Röntgenverlaufskontrolle mit zunehmender Konsolidierung des Distraktionskallus. fBeispiel eines Verfahrenswechsels mit Stabilisierung der Elle bzw. des Distraktionskallus mittels Plattenosteosynthese (z. B. Ulnaosteotomieplatte der Fa. Synthes)

Komplikationen, die unmittelbar mit bzw. nach dem operativen Eingriff auftreten können. Erwähnenswert sind hierbei die Nervenverletzungen, die alle 3 Nerven im Ellenbogenbereich betreffen können. Führend - wie bei der Primärversorgung - sind der N. ulnaris und durch die oftmals notwendige Freilegung und Manipulation der N. radialis. Dieser ist durch die damit verbundene Fallhand besonders belastend für die weitere Betreuung in der Behandlung. Auch Einzelfälle mit Perfusionsstörungen und Kompartmentsyndromen werden beschrieben. Abhängig von Alter und Osteosyntheseverfahren finden sich spezifische Probleme. Beim Gebrauch des Fixateurs sind die Pininfektion und die unzureichende Konsolidierung erwähnenswert, die z.B. zu Refrakturen oder erneuter Deformität führen kann. Dies gilt insbesondere bei der Monteggia-Läsion, wo eine Kallusdistraktion im Unterarmbereich notwendig ist und auch kritische deformitätsbedingte Anpassungen eine Reluxation und ein Therapieversagen mit verursachen können. Durch vorbestehende Veränderungen am Radiuskopf und die notwendigen Manipulationen zur Gelenkeinstellung mit dem Risiko von Weichteilverkalkungen, Osteonekrosen und Ossifikationen kann sich eine Synostose ausbilden, die zur Einschränkung der Unterarmdre- hung führt. Auch die Auswirkung auf das Handgelenk ist zu betonen und die ggf. sekundäre Korrektur (Nivellierung eines relativen Ellenvorschubs) in diesem Bereich. Ebenso muss die Pseudarthrosebildung nach Korrektur des distalen Humerus oder gar Repseudarthrose nach Revision des Condylus radialis erwähnt werden. Trotz gutem Heilungsverlauf und kosmetisch ansprechendem Ergebnis bleibt die Bewegungseinschränkung die häufigste Komplikation und ist $\mathrm{z}$. T. 5-fach häufiger als die zuvor erwähnten Probleme [10, 13, 16, 39-41]. 
Tab. 4 Merkmale der kongenitalen Dislokation [42]

\begin{tabular}{|c|c|}
\hline Anatomisches Areal & Merkmale \\
\hline Capitulum & Hypoplasie \\
\hline Ulna & $\begin{array}{l}\text { Verkürzt, ventraler Bogen bei ventraler, dorsaler Bogen bei } \\
\text { dorsaler Dislokation }\end{array}$ \\
\hline Radius & $\begin{array}{l}\text { Domförmig bei ventraler, verlängert/schmal bei dorsaler } \\
\text { Dislokation }\end{array}$ \\
\hline Radiuskopf & $\begin{array}{l}\text { Luxationsrichtung } 43 \% \text { dorsal, } 47 \% \text { ventral, } 10 \% \text { radiale } \\
\text { Luxation }\end{array}$ \\
\hline Ellenbogen der Gegenseite & Häufig bilateral \\
\hline Sonstiges Areal, z. B. Hand & Kombination mit anderen Anomalien/Syndromen \\
\hline
\end{tabular}

Tab. 5 Auflistung der Folgeveränderungen nach chronischer Monteggia-Läsion

\begin{tabular}{l|l}
\hline Anatomisches Areal & Sekundärdeformität \\
\hline Radiuskopf & Konvexer Radiuskopf \\
\hline $\begin{array}{l}\text { Proximaler Radius } \\
\text { Proximale Ulna }\end{array}$ & $\begin{array}{l}\text { Achsabweichung z. B. Radiushals } \\
\text { Deformität mit radialer Konvexität, Ausweitung der proxima- } \\
\text { len Elle über das Capitulum }\end{array}$ \\
\hline $\begin{array}{l}\text { Deformität PRUG (proximales } \\
\text { Radioulnargelenk) }\end{array}$ & Neogelenk, Synostose \\
\hline
\end{tabular}

\section{Fazit für die Praxis}

- Die operative Therapie von posttraumatischen Deformitäten am Ellenbogen ist aufgrund der Komplexität der Gelenkanatomie und der dort befindlichen Wachstumsfugen eine besondere Herausforderung.

- Entscheidend bleibt die adäquate Therapie bei der akuten Verletzung.

- Kommt es dennoch zu einer posttraumatischen Deformität, muss eine differenzierte Indikationsstellung zur Korrektur erfolgen. Dazu gehört, bei funktionellen Störungen ein erreichbares Ziel abzuschätzen und die bereits erfolgte Anpassung der verschiedenen beteiligten Strukturen bzw. Gelenke an die Deformität zu berücksichtigen.

- Unter kosmetischen Aspekten und aufgrund der zum Teil hohen Komplikationsrate muss die Indikation sehr kritisch gestellt werden.

- Bei der chronischen Radiuskopfluxation nach Monteggia-Läsion muss zwischen kongenitalen bzw. entwicklungsbedingten Deformitäten differenziert und eher eine zurückhaltende Therapie propagiert werden. Dies erfordert eine ausführliche Besprechung mit Kind bzw. Eltern.

\section{Korrespondenzadresse}

\section{Prof. Dr. J. Frank} lungschirurgie, Universitätsklinikum Frankfurt, Goethe-Universität

Theodor-Stern-Kai 7, 60590 Frankfurt am Main, Deutschland

j.frank@trauma.uni-frankfurt.de

\section{Einhaltung ethischer Richtlinien}

Interessenkonflikt. J. Frank, A.L. Sander, M. Voth und I. Marzi geben an, dass kein Interessenkonflikt besteht.

Dieser Beitrag beinhaltet keine von den Autoren durchgeführten Studien an Menschen oder Tieren.

The supplement containing this article is not sponsored by industry.

\section{Literatur}

1. Kraus R (2013) The pediatric vs. the adolescent elbow. Some insight into age-specific treatment. Eur J Trauma Emerg Surg 40:15-22. doi:10.1007/ s00068-013-0342-y

2. von Laer L (1986) Frakturen und Luxationen im Wachstumsalter. Thieme, Stuttgart

3. Marzi I (2009) Kindertraumatologie, 2. Aufl. Springer, Berlin, Heidelberg

4. Marzi I (2016) Kindertraumatologie, 3. Aufl. Springer, Berlin, Heidelberg

5. Weinberg AM, Marzi I, Günter SM et al (2002) Die suprakondyläre Oberarmfraktur im Kindesalter eine Effizienzstudie Ergebnisse der multizentrischen Studie der Sektion Kindertraumatologieder
Klinik für Unfall-, Hand- und Wiederherstel-
Deutschen Gesellschaft für Unfallchirurgie - Teil I: Epidemiologie, Effektivitätsprüfung und Klassifikation. Unfallchirurg 105:208-216. doi:10.1007/ s001130100314

6. Hasler CC (2001) Supracondylar fractures of the humerus in children. Eur J Trauma 27:1-15. doi:10. 1007/s00068-001-1088-5

7. von Laer L, Pirwitz A, Vocke AK (1997) Posttraumatic problem cases involving the elbow in children. Orthopäde 26:1030-1036

8. Weise K, Schwab E, Scheufele TM (1997) Ellenbogenverletzungen im Kindesalter. Unfallchirurg 100:255-269. doi:10.1007/s001130050117

9. Weinberg AM, Reilmann H (2000) Frakturen der Diaphyse im Kindesalter Teil II: Obere Extremität. Unfallchirurg 103:144. doi:10.1007/ s001130050026

10. Kim HT, Lee JS, Yoo Cl (2005) Management of cubitus varus and valgus. J Bone Joint Surg Am 87:771-780. doi:10.2106/JBJS.D.01870

11. French PR (1959) Varus deformity of the elbow following supracondylar fractures of the humerus in children. Lancet 2:439-441

12. Raney EM, Thielen Z, Gregory S, Sobralske M (2012) Complications of supracondylar osteotomies for cubitus varus. J Pediatr Orthop 32:232-240. doi:10. 1097/BPO.0b013e3182471d3f

13. Kumar K, Sharma VK, Sharma R, Maffulli N (2000) Correction of cubitus varus by French or dome osteotomy: a comparative study. J Trauma 49:717-721

14. Bellemore MC, Barrett IR, Middleton RW et al (1984) Supracondylar osteotomy of the humerus for correction of cubitus varus. J Bone Joint Surg Br 66:566-572

15. Hui JHP, Torode IP, Chatterjee A (2004) Medial approach for corrective osteotomy of cubitus varus: a cosmetic incision. J Pediatr Orthop 24:477-481

16. Koch PP, Exner GU (2003) Supracondylar medial open wedge osteotomy with external fixation for cubitus varus deformity. J Pediatr Orthop B 12:116-122. doi:10.1097/01.bpb.0000049571. 52224.c8

17. Karatosun V, Alekberov C, Alici E et al (2000) Treatment of cubitus varus using the llizarov technique of distraction osteogenesis. J Bone Joint Surg Br 82:1030-1033

18. Horstmann HM, Blyakher AA, Quartararo LG, Cavalier R (2000) Treatment of cubitus varus with osteotomy and llizarov external fixation. Am J Orthop 29:389-391

19. Horst M, Altermatt S, Weber DM et al (2010) Pitfalls of lateral external fixation for supracondylar humeral fractures in children. Eur J Trauma Emerg Surg 37:405-410. doi:10.1007/s00068-010-00625

20. Linhart WE, Kraus T (2008) Reconstruction of humeroradialjoint. Orthop Traumatol 20:396-408. doi:10.1007/s00064-008-1411-y

21. Bado JL (1967) The Monteggia lesion. Clin Orthop Relat Res 50:71-86

22. Letts M, Locht R, Wiens J (1985) Monteggia fracture-dislocations in children. J Bone Joint Surg Br67:724-727

23. Josten C, Freitag S (2008) Monteggia and Monteggia-like-lesions: classification, indication, and techniques in operative treatment. Eur J Trauma Emerg Surg 35:296-304. doi:10.1007/ s00068-008-8028-6

24. Kemnitz S, De Schrijver F, De Smet L (2000) Radial head dislocation with plastic deformation of the ulna in children. A rare and frequently missed condition. Acta Orthop Belg 66:359-362 
25. Olney BW, Menelaus MB (1989) Monteggia and equivalent lesions in childhood. J Pediatr Orthop 9:219-223

26. Slongo TF (2008) Correction osteotomy of neglected "Monteggia" lesion with an external fixator. Orthop Traumatol 20:435-449. doi:10. 1007/s00064-008-1414-8

27. Stoll TM, Willis RB, Paterson DC (1992) Treatment of the missed Monteggia fracture in the child. J Bone Joint Surg Br 74-B:436-440

28. Best TN (1994) Management of old unreduced monteggia fracture dislocations of the elbow in children.J Pediatr Orthop 14:193

29. Tajima T, Yoshizu T (1995) Treatment of longstanding dislocation of the radial head in neglected Monteggia fractures. J Hand Surg Am 20:S91-S94

30. Ring D, Waters PM (1996) Operative fixation of Monteggia fractures in children. J Bone Joint Surg Br 78-B:734-739

31. von Laer L, Pirwitz A, Hasler CC (2000) Late missed Monteggia fractures. Tech Orthop 15:27

32. Gyr BM, Stevens PM, Smith JT (2004) Chronic Monteggia fractures in children: outcome after treatment with the Bell-Tawse procedure. J Pediatr Orthop B 13:402

33. Degreef I, De Smet L (2004) Missed radial head dislocations in children associated with ulnar deformation: treatment by open reduction and ulnar osteotomy. J Orthop Trauma 18:375-378

34. von Laer L, Hasler C, Hell-Vocke AK (2005) Late missed Monteggia lesions - reconstruction of the humeroradial joint. Eur J Trauma 31:597-607. doi:10.1007/s00068-005-6605-5

35. Hasler CC, von Laer L, Hell AK (2005) Open reduction, ulnar osteotomy and external fixation for chronic anterior dislocation of the head of the radius. J Bone Joint Surg Br 87-B:88-94. doi:10. 1302/0301-620X.87B1.14669

36. Lädermann A, Ceroni $D$, Lefèvre $Y$ et al (2007) Surgical treatment of missed Monteggia lesions in children. J Child Orthop 1:237-242. doi:10.1007/ s11832-007-0039-z

37. Bhaskar A (2013) Current concepts in the management of missed Monteggia fracturedislocation. Curr Orthop Pract 24:49-52. doi:10. 1097/BCO.0b013e31827ddc42

38. Korner J, Hansen M, Weinberg A et al (2004) Monteggia fractures in childhood? Diagnosis and management in acute and chronic cases. Eur J Trauma 30:361-370. doi:10.1007/500068-0041343-7

39. Kraus T, Tauber S, Linhart W (2013) Posttraumatic complications on children's elbows. Orthopäde 42:57-70. doi:10.1007/s00132-012-2029-1

40. Usui M, Ishii S, Miyano S et al (1995) Threedimensional corrective osteotomy for treatment of cubitus varus after supracondylar fracture of the humerus in children. J Shoulder Elbow Surg 4:17-22

41. Nakamura K (2009) Long-term clinical and radiographic outcomes after open reduction for missed Monteggia fracture-dislocations in children. J Bone Joint Surg Am 91:1394. doi:10. 2106/JBJS.H.00644

42. Morrey BF, Sanchez-Sotelo J (2009) The elbow and its disorders, 4. Aufl. Saunders, Philadelphia 